\title{
BeppoSAX confirmation of beamed afterglow emission from GRB 990510
}

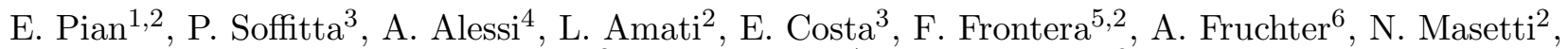 \\ E. Palazzi ${ }^{2}$, A. Panaitescu ${ }^{7}$, and P. Kumar ${ }^{8}$ \\ 1 Osservatorio Astronomico di Trieste, Via G.B. Tiepolo 11, 34131 Trieste, Italy \\ 2 Istituto Tecnologie e Studio Radiazioni Extraterrestri, CNR, Via Gobetti 101, 40129 Bologna, Italy \\ 3 Istituto di Astrofisica Spaziale, CNR, via Fosso del Cavaliere, Area di Ricerca Tor Vergata, 00133 Rome, Italy \\ 4 Dipartimento di Astronomia, Università di Bologna, Via Ranzani 1, 40127 Bologna, Italy \\ 5 Dipartimento di Fisica, Università di Ferrara, Via Paradiso 11, 44100 Ferrara, Italy \\ 6 Space Telescope Science Institute, 3700 San Martin Drive, Baltimore, MD 21218, USA \\ 7 Department of Astrophysical Sciences, Princeton University, NJ 08544, USA \\ 8 Institute for Advanced Study, Olden Lane, Princeton, NJ 08540, USA
}

Received 6 December 2000 / Accepted 26 March 2001

\begin{abstract}
We compare the prompt X-ray (2-10 keV) emission of GRB 990510 measured by the BeppoSAX Wide Field Cameras (WFC) during the burst to the X-ray afterglow detected by the BeppoSAX Narrow Field Instruments. A single power-law model for the afterglow, $f(t) \propto t^{-1.42}$, is ruled out. Provided the initial time of the afterglow is assumed to coincide with the last short pulse in the X-ray prompt event (i.e., $72 \mathrm{~s}$ after the GRB trigger time), the X-ray emission from $\sim 80$ to $10^{5} \mathrm{~s}$ after the GRB trigger is well described by an external shock expanding in a decelerating jet, in which synchrotron radiation takes place. This model, represented by a double power-law of indices $\alpha_{1} \simeq 1$ and $\alpha_{2} \simeq 2$ before and after a jet collimation break time of $\sim 0.5$ days after GRB, respectively, is consistent with the second and third upper limits measured by the WFC, but not with the first. This may be related to inhomogeneities in the circumburst medium. Our finding indicates that the temporal behavior of the GRB 990510 X-ray afterglow is similar to that at optical wavelengths, and thus strengthens the interpretation of the multiwavelength afterglow as synchrotron emission in a jet with decreasing Lorentz factor. GRB 990510 is thus the only burst in which evidence of a spreading jet has been found in X-rays.
\end{abstract}

Key words. gamma rays: bursts - X-rays: general - radiation mechanisms: non-thermal

\section{Introduction}

GRB 990510 was detected by the CGRO BATSE instrument and by the Gamma-Ray Burst Monitor (GRBM) onboard BeppoSAX on 1999 May 10.3674 UT. Following the localization by the BeppoSAX Wide Field Cameras (WFC, 2-26 keV) Unit 2, the afterglow search promptly started and allowed the detection of a bright and variable source at X-ray, optical and radio wavelengths (Kuulkers et al. 2000, hereafter KAK00; Harrison et al. 1999; Stanek et al. 1999; Israel et al. 1999). Polarimetry of the optical transient yielded the first measurement of an afterglow polarization percentage $(1.7 \%$, Wijers et al. 1999; Covino et al. 1999). Optical spectroscopy detected many absorption lines and led to the estimate of a lower limit on the redshift, $z>1.62$ (Vreeswijk et al. 2001). Late epoch photometry from ground and with HST put limits on the mag-

Send offprint requests to: E. Pian,

e-mail: pian@tesre.bo.cnr.it nitude of the host galaxy (Beuermann et al. 1999; Fruchter et al. 1999) and eventually provided evidence for a faint, small nebulosity (Fruchter et al. 2000; Bloom 2000). The optical and radio temporal behavior of the afterglow suggests that this has developed within a jet progressively spreading and laterally expanding (Harrison et al. 1999; Stanek et al. 1999). Panaitescu \& Kumar (2000, hereafter PK00) have modeled the afterglow multiwavelength light curves with synchrotron radiation in a relativistic jet expanding in a medium of uniform density.

The signature of a decreasingly collimated jet is the simultaneous steepening of afterglow light curves at all frequencies (Sari et al. 1999; Rhoads 1999; see however Wei \& $\mathrm{Lu} \mathrm{2000)}$. This is clearly seen in the optical data of GRB 990510, which can be described by two temporal power-laws, $f(t) \propto t^{-\alpha}$, of indices $\alpha \sim 0.8$ and $\alpha \gtrsim 2$ before and after a break occurred at approximately 1 day after the GRB, respectively. Instead, the X-ray afterglow data are not critical in discerning between a spherical 


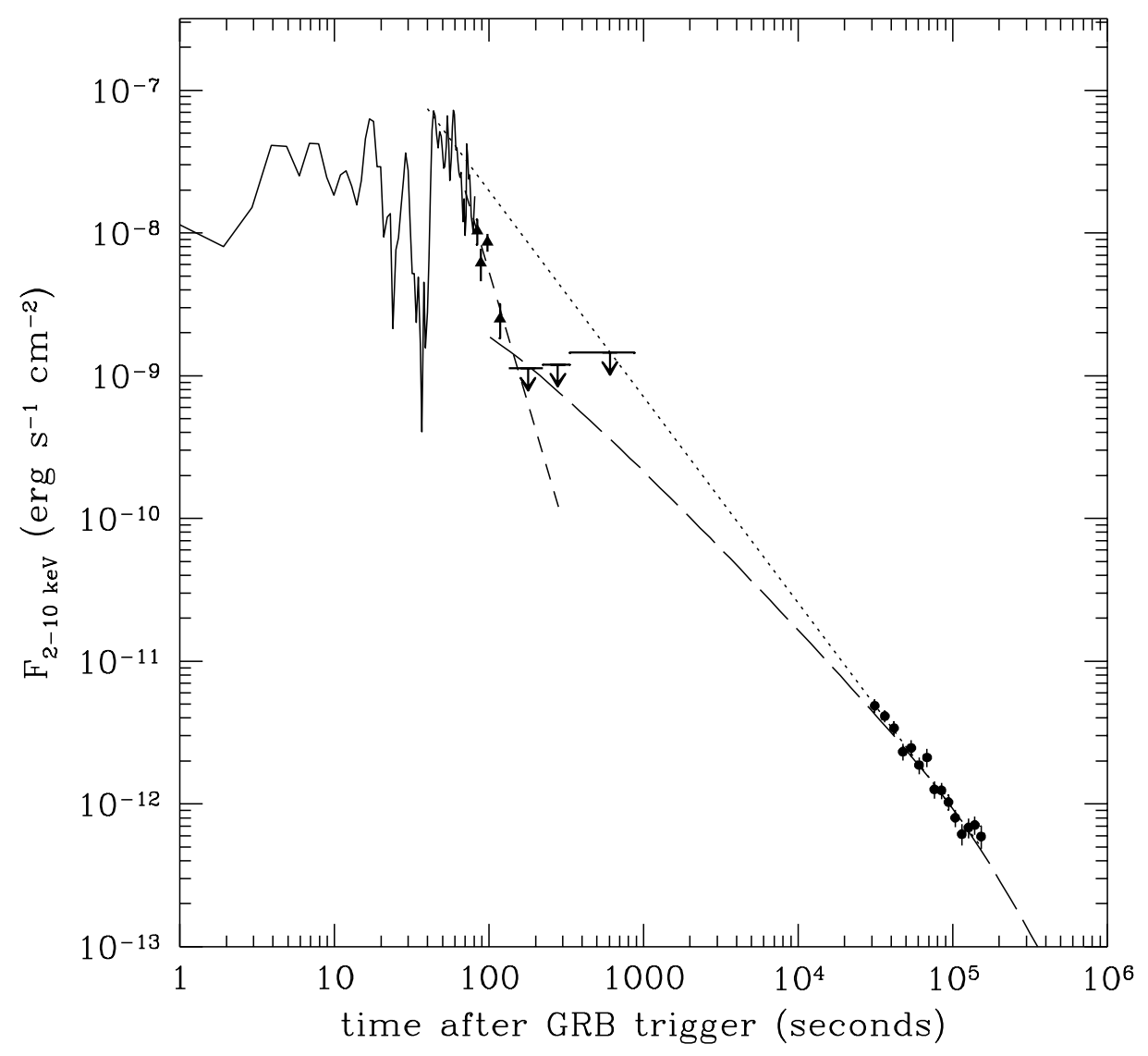

Fig. 1. BeppoSAX light curves of GRB 990510 in the 2-10 keV range: WFC temporal profile (solid curve, filled triangles and $3-\sigma$ upper limits) and afterglow NFI measurements (filled circles). Also shown are the single power-law $f(t) \propto t^{-1.42}$ (dot) fitted to the afterglow by KAK00, the power-law $f(t) \propto t^{-3.7}$ (short dash) which best-fits the last four points of the WFC profile, and the double power-law of indices $\alpha_{1} \simeq 1$ and $\alpha_{2} \simeq 2$ (long dash) derived from PK00 afterglow model $(z=1.62$. The fit has $\chi^{2}=41$ for 69 degrees of freedom). The six model parameters are isotropic equivalent energy $E_{0}=5 \times 10^{53} \mathrm{erg}$, initial jet half-opening angle $\theta_{0}=2.7$ degrees, density of homogeneous external medium $n=0.13 \mathrm{~cm}^{-3}$, fraction of shock energy going into electrons $\epsilon_{\mathrm{e}}=4.6 \times 10^{-2}$, magnetic energy density $\epsilon_{B}=9 \times 10^{-4}$, exponent of injected power-law electron distribution $p=2$. The amount of jet energy lost radiatively until 2 days after GRB is $45 \%$. Note that these parameters, slightly revised with respect to those reported in PK00 for this burst, are affected by rather large uncertainties, and must therefore only be considered as representative. The extrapolation of the single power-law $t^{-1.42}$ backward to the time of the prompt event is not consistent with the WFC latest points and upper limits, while the double power-law matches well the last WFC point and upper limits.

or jet hydrodynamical expansion (KAK00), due to limited signal-to-noise ratio of the BeppoSAX Narrow Field Instruments (NFI) data, to the relatively long interval elapsed between GRB detection and NFI pointing $(8 \mathrm{hr})$, and to the limited duration of the observation $(36 \mathrm{hr})$. The data are consistent both with a single power-law $f(t) \propto t^{-1.42}$ and with a double power-law. Based on the standard afterglow synchrotron model, KAK00 argue that if decreasing beaming is taking place, a break should occur at $\sim 1$ day after the GRB, as in the optical, and the indices of the temporal power-laws should be $\sim 1.1$ before the break, i.e. steeper than in the optical by $\Delta \alpha \simeq 0.25$, and $\sim 2.1$ after the break, i.e., equal to the optical (see Sari et al. 1998; Sari et al. 1999). By applying these parameters to the X-ray light curve, KAK00 find a good agreement.

Since the two descriptions of the X-ray afterglow light curve (single or double power-law) would imply a very different flux level at the early epochs (from few to hun- dreds of seconds after the GRB), a crucial test to select the correct model is provided by the comparison of the $\mathrm{X}$ ray afterglow NFI data and the X-ray prompt event WFC data. Moreover, simultaneous multiwavelength spectra of the afterglow at many epochs would allow us to follow its temporal evolution and specifically to map the behavior of the synchrotron cooling frequency, which is critical in distinguishing an isotropic from a beamed expansion. Therefore, we have retrieved from the BeppoSAX archive and analyzed the WFC data of the prompt event, and we have collected from the literature simultaneous optical and X-ray data of the afterglow of GRB 990510. Our WFC data analysis is described in Sect. 2, and the results are compared to the X-ray NFI afterglow data in Sect. 3. The multiwavelength data analysis and results are reported in Sect. 4. The X-ray and multiwavalength findings are discussed in Sect. 5, and a summary is given in Sect. 6 . 


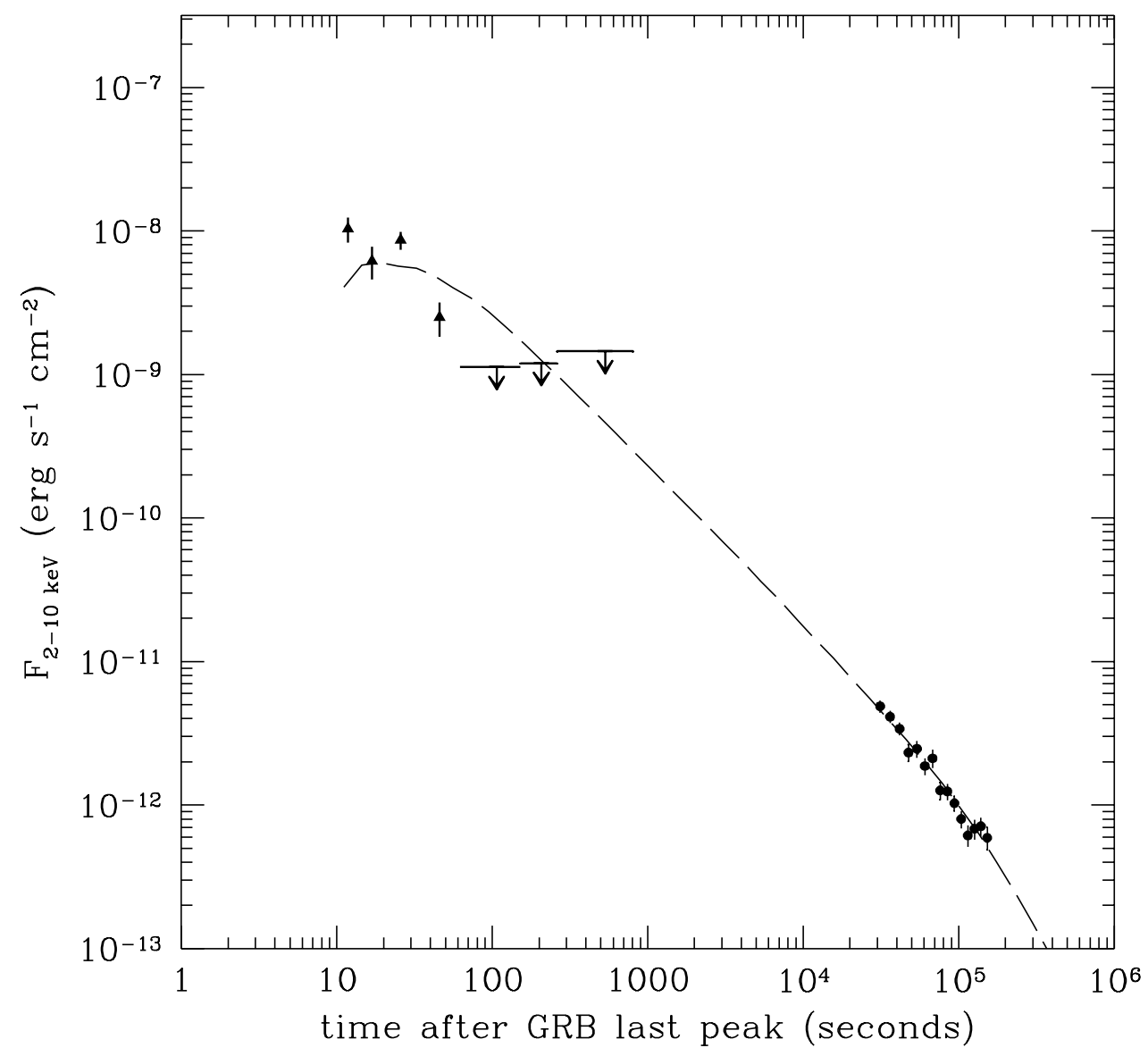

Fig. 2. BeppoSAX WFC (last four measurements and three upper limits) and NFI light curves of GRB 990510 in the $2-10 \mathrm{keV}$ range, as in Fig. 1, but with assumed initial time equal to the time of the last GRB peak (i.e., $72 \mathrm{~s}$ after trigger, see Fig. 1). Overimposed is the curve obtained by fitting the PK00 model to the multiwavelength afterglow data, plus the 4 last WFC points. The downward bending of the model curve toward the earliest epochs is due to the fact that the observer frame deceleration timescale of the blast implied by the model parameters is $\sim 6 \mathrm{~s}$, so that X-ray emission starts rising only after this time.

\section{Wide Field Camera data analysis and results}

The WFC data of the prompt GRB 990510 event have been retrieved from the BeppoSAX archive (see also Briggs 2000) and analyzed by means of a standard package which includes the "iterative removal of source" procedures (IROS, V. 105.108, e.g. Jager et al. 1997). These implicitly subtract the background and the other sources in the field of view. The burst direction was offset by $15^{\circ}$ with respect to the WFC axis, which implies an effective collecting area of $32 \mathrm{~cm}^{2}$. We have analyzed the spectrum measured by the WFC during the burst using the XSPEC package (V. 10.0, Arnaud 1996), and obtained a good fit with a power-law $f(\nu) \propto \nu^{-\beta}$ of in$\operatorname{dex} \beta=0.23 \pm 0.08$ (absorbed by Galactic neutral hydrogen, $N_{\mathrm{HI}}=0.94 \times 10^{21} \mathrm{~cm}^{-2}$, see also KAK00). We used this spectral shape to convert the WFC count rates to fluxes. The burst fluence in the $2-10 \mathrm{keV}$ energy range is $(3.3 \pm 0.1) \times 10^{-6} \mathrm{erg} \mathrm{cm}^{-2}$.

Figure 1 shows the temporal profile of the prompt event and of the X-ray afterglow measured by the NFI during the interval from 8 to 44 hours after the burst in the energy range $2-10 \mathrm{keV}$. Significant WFC signal is de- tected up to $\sim 130 \mathrm{~s}$ after the GRB trigger. The four last points of the WFC profile (encompassing the interval from 80 to $130 \mathrm{~s}$ after GRB and shown as filled triangles in Fig. 1) indicate a fast decay, best-fitted with a temporal power-law of index $\alpha=3.7 \pm 1.5$. The spectrum measured by the WFC during this time interval is well fitted with a power-law of slope $\beta=0.96 \pm 0.26$. This is consistent with the upper limits provided by the BeppoSAX GRBM (40-700 keV) in the same time interval. In Fig. 1 are also reported the 3- $\sigma$ upper limits on the X-ray flux, determined by the WFC after the GRB subsided under background.

\section{Comparison of the prompt and afterglow X-ray emission}

The tail of the X-ray prompt emission, as measured by the WFC, and the following upper limits are inconsistent with an extrapolation of the single power-law fitted by KAK00 to the afterglow back to the time of the burst, as shown in Fig. 1. Therefore the X-ray afterglow can not follow a single power-law, and may instead have a shallower decay 
at earlier times and then continuously steepen, similar to the optical behavior. To test this hypothesis and to predict the X-ray afterglow flux at the early epochs after the GRB ( 100 s), we have adopted the model of PK00, which accounts well for the multiwavelength afterglow emission of GRB 990510 (see their Fig. 3).

A fit of the model to the NFI X-ray, optical $V$ and $I$ band, and $8.5 \mathrm{GHz}$ radio data of the afterglow (i.e., past $10^{4} \mathrm{~s}$ after trigger) yields a $\chi^{2}=41$ for a total of 69 degrees of freedom. The fit parameters are reported in the caption to Fig. 1. The WFC X-ray measurements and upper limits have not been included in the fit, because we have made the hypothesis that the early X-ray emission is due to other mechanisms, so that the basic modeling assumption, that a self-similar outflow has set in, may not be satisfied.

The model curve asymptotically tends, at early and late epochs, to power-laws of indices $\alpha_{1} \sim 1$ and $\alpha_{2} \sim 2$, respectively, with an achromatic collimation break time of $\sim 0.5$ days, compatible with that empirically determined by Stanek et al. (1999), Harrison et al. (1999), Israel et al. (1999). The temporal indices are consistent with those suggested by KAK00 for the X-ray afterglow in a beamed scenario, and in fact the curve reproduces well the trend of the X-ray afterglow data. A comparison to the prompt $\mathrm{X}$-ray data shows that the afterglow model curve lies below the four latest WFC measurements, but in excellent agreement with the last of them and with the three WFC upper limits (Fig. 1).

However, while this match in flux between the observed last point of the X-ray prompt event and the predicted afterglow at that epoch is very good (and supported by the WFC upper limits), the change in temporal slope between the tail of the prompt event and the early afterglow is large (from $\alpha \sim 3.7$ to $\alpha \sim 1$ ). Therefore, we have considered the possibility that this abrupt change is only determined by the choice of the initial time, and we have assumed that the X-ray afterglow starts at the time of the last peak of the WFC profile, occurring at $72 \mathrm{~s}$ after the GRB trigger time (see Fig. 1). This assumption is more physical, because one expects that different pulses in a GRB light curve are produced by different successive shocks, and that the afterglow sets in after the last one. Under this assumption, the power-law index of the last 4 WFC points temporal decay is $\alpha=0.9 \pm 0.2$ (with a reduced $\chi_{\nu}^{2}=4.5$, due to the high scatter of the points). In Fig. 2 we report the last 4 WFC points, the 3 upper limits and NFI data at epochs computed with respect to the GRB last peak. We have re-fitted the PK00 model to the multiwavelength data, by adding this time the 4 last WFC fluxes (but not the 3 upper limits), which are now assumed to be part of the afterglow, and therefore produced by an external shock. The fit has $\chi^{2}=50$ for 73 degrees of freedom, and the model curve is reported in Fig. 2, superimposed onto the data. The new fit parameters are very close to the previous ones, obtained by assuming a same initial time for GRB and afterglow, and by excluding the 4 latest WFC measurements. The model curve accounts well for all WFC and NFI data, except the first of the 3 WFC upper limits, which lies below it by a factor $\sim 2$.

\section{The broad-band spectrum}

In a spreading jet scenario the synchrotron cooling frequency, $\nu_{\mathrm{C}}$, is predicted to decrease before the occurrence of the temporal break at which the light curve starts steepening, because till then the observed evolution does not differ from a spherically symmetric case (the beaming angle is still much smaller than the jet opening angle), and to stabilize after the break to a constant value (Sari et al. 1998; Sari et al. 1999). To test this temporal behavior, we have compared the X-ray and optical data of the GRB 990510 afterglow: its simultaneous X-ray and optical light curves are shown in Fig. 3a. The host galaxy contribution (which is very small, $V \approx 28$, Fruchter et al. 2000 ; Bloom 2000) has not been subtracted from the optical data. We have selected seven epochs at which simultaneous measurements in X-rays and in at least 3 optical bands are available. The simultaneity criterion was a time separation between the optical and X-ray sampling of less than $20 \mathrm{~min}$; only for May 11.978 the separation is $1.3 \mathrm{hr}$, which we have verified to be larger than the time scale for significant optical variability at that epoch. We note that, since $\nu_{\mathrm{C}}$ is predicted to be well above the $B$ optical band (PK00), the slight curvature of the optical spectrum observed by Stanek et al. (1999) and Beuermann et al. (1999) in this band cannot be ascribed to synchrotron cooling, and may be due instead to intrinsic or intervening absorption. Therefore, for consistency we excluded the $B$ band data from the following fit and computation. After dereddening the optical data for the Galactic absorption using $E_{B-V}=0.2 \mathrm{mag}$ and the extinction curve of Cardelli et al. (1989), converting them to fluxes with the zero points of Fukugita et al. (1995), and rescaling them, via interpolation, to the time of the X-ray measurement, we estimated $\nu_{\mathrm{C}}$ at each epoch by intersecting the optical spectral slope (evaluated through a power-law fit of the VRI data) and the X-ray spectral slope, as reported by KAK00 in their Table 3. The resulting values are reported in Fig. $3 \mathrm{~b}: \nu_{\mathrm{C}}$ is approximately $7 \times 10^{15} \mathrm{~Hz}$ and does not exhibit significant variability, given the large errors.

\section{Discussion}

The prompt (1-70 s after trigger) X-ray emission of GRB 990510 exhibits erratic behavior (Fig. 1), suggestive of internal shocks occurring in an unsteady wind, rather than an external shock propagating in the circumburst medium.

Most of the GRB X-ray afterglows observed by BeppoSAX are well accounted for by isotropic synchrotron radiation in an external shock and described by a single power-law decay of index $\alpha \sim 1.1-1.6$ which connects smoothly with the tail of the X-ray prompt emission detected by the WFC simultaneously with the GRB 


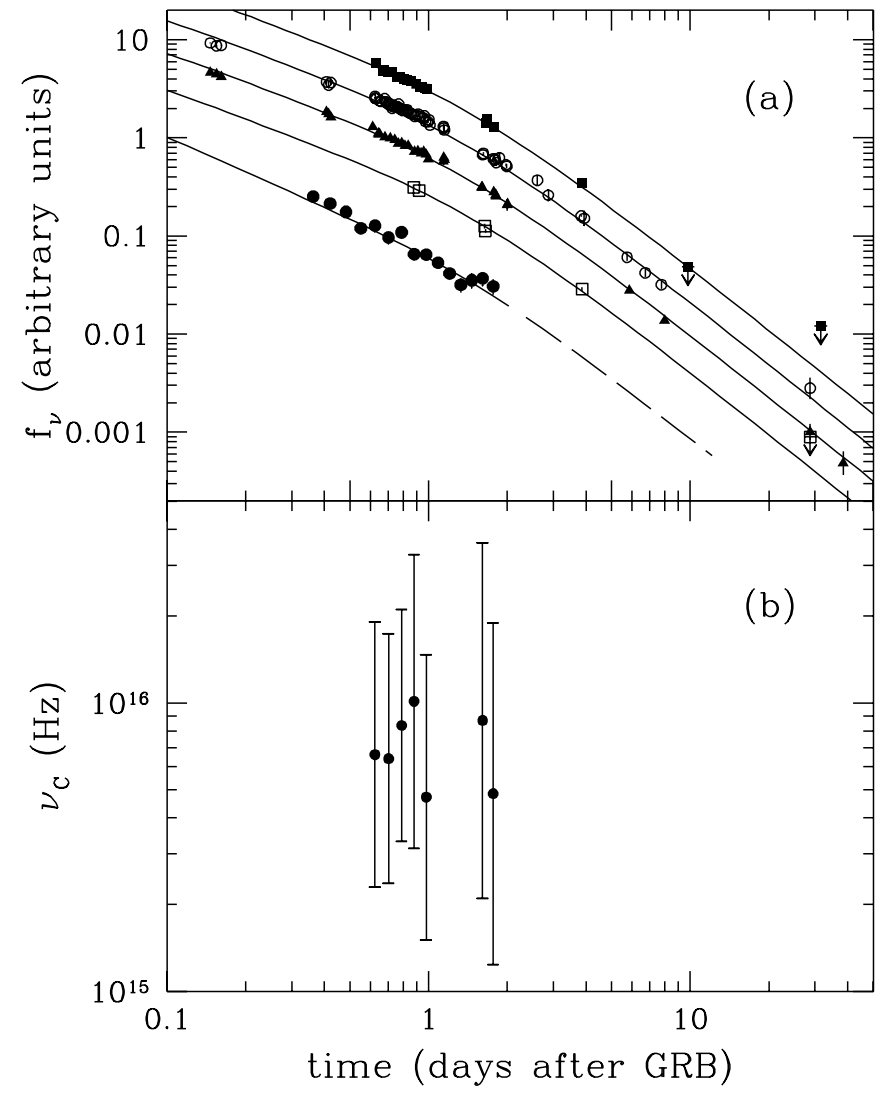

Fig. 3. a) Simultaneous X-ray and optical light curves of the GRB 990510 afterglow. Optical data in $I$ (filled squares), $R$ (empty circles), $V$ (filled triangles) and $B$ (open squares) filters, and X-ray data at $5 \mathrm{keV}$ (filled circles) have been collected from Harrison et al. (1999), Stanek et al. (1999), Israel et al. (1999), Beuermann et al. (1999), Fruchter et al. (1999), KAK00. The flux normalization is in arbitrary units, except for the X-ray data, which are reported in $\mu \mathrm{Jy}$. Superimposed on the data are the curves (solid) corresponding to the model of PK00. The model curve relative to the X-ray data is extrapolated up to 10 days after GRB (dashed). The model parameters are reported in the caption to Fig. 1. b) Synchrotron cooling frequency, estimated from simultaneous optical and Xray data, as a function of time.

(Costa et al. 1997; Piro et al. 1998; in 't Zand et al. 1998; Nicastro et al. 1999; Costa 1999). This suggests that generally the latest X-rays detected during a GRB already represent afterglow emission (Sari \& Piran 1999; Frontera et al. 2000).

In GRB 990510, although a single power-law $f(t) \propto$ $t^{-1.42}$ fits well the X-ray afterglow data $\left(3 \times 10^{4}-2 \times 10^{5} \mathrm{~s}\right.$ after trigger, KAK00), its extrapolation backward to the time of the burst exceeds the flux of the WFC last four points and upper limits (Fig. 1). Therefore the X-ray afterglow emission fall-off until at least $1000 \mathrm{~s}$ must have been shallower than at 1 day, i.e. there was a break in the X-ray emission prior to or during the NFI measurements. This break can be due either 1) to the passage of a spectral break, either injection or cooling, in a spherical symmetry, or 2) to collimation of ejecta. Assuming case (1), the first WFC upper limit and the first NFI data require that the X-ray decay prior to the spectral break passage is shallower than $t^{-1}$. It is unlikely that the injection break passes through the X-ray band after $1000 \mathrm{~s}$, as this would require $\epsilon_{\mathrm{e}}$ and $\epsilon_{B}$ to be close to equipartition, and a large energy $E_{0}$ (then the cooling frequency would be well below injection, and the X-ray light-curve would decay as $t^{-1 / 4}$ prior to the passage of the injection break, and as $t^{-(3 \mathrm{p}-2) / 4}$ thereafter $)$. On the other hand, the steepening of the light-curve decay from shallower than $t^{-1}$ to $t^{-1.4}$ exceeds the maximum index increase of $1 / 4$ that the cooling break passage can produce. Thus, the only remaining possibility is case (2), i.e., that the ejecta are collimated. Furthermore, the break seen in the optical data also argues in favor of the collimation. Synchrotron radiation in a shock developing in a jet which decelerates in a uniform medium accounts well for the X-ray afterglow emission, is consistent with the last WFC measurement, and satisfies the constraints imposed by the three WFC upper limits (Fig. 1).

The transition from the temporal decline rate in the last phase of the burst and the early afterglow as predicted by the model is not smooth. The final part of the WFC profile (from 80 to $130 \mathrm{~s}$ after the GRB) is decreasing much more fastly $\left(t^{-3.7}\right)$ than the predicted afterglow during the same interval and soon thereafter, and therefore cannot be reconciled with it (Sari \& Piran 1999). An explanation may be that, even if the afterglow of GRB 990510 may start earlier than $\sim 130$ s after GRB, till then the emission is dominated by internal shocks. This early X-ray decline can be steeper than $2+\beta$, where $\beta$ is the spectral index of the GRB X-ray emission (see Fig. 1 in Kumar \& Panaitescu 2000). When the external shock starts dominating the emission, the X-ray spectral index is expected to assume the value $\beta=p / 2 \simeq 1$, consistent with the X-ray spectral slope measured by KAK00 for the afterglow. However, we measure an index $\beta \sim 1$ also for the WFC spectrum during the last $50 \mathrm{~s}$ of the prompt event (i.e., from 80 to $130 \mathrm{~s}$ after trigger), which strongly suggests that the GRB X-ray tail already represents afterglow emission (see e.g. the case of GRB 970228, Frontera et al. 1998).

The mismatch between the temporal decay of the observed last GRB phase and the predicted early afterglow in GRB 990510 may be removed by assuming that the X-ray afterglow starts at the last GRB peak, observed by the WFC at $72 \mathrm{~s}$ after the instrumental trigger time. As noted by Giblin et al. (1999) for GRB 980923, there is no reason why the afterglow start time should coincide with the time of the trigger. In fact, the internal shock GRB emission and external shock afterglow emission are physically different and may be independent in timing (see also GRB 920723, Burenin et al. 1999; GRB 910402, Tkachenko et al. 2000). The new assumption of initial time allows us to describe with an external shock model also the latest X-ray emission of the GRB, more in line with observations of many other GRBs. Only the first of the WFC upper limits is clearly inconsistent with the model (see Fig. 2). Rather than considering this as an evidence 
against a same mechanism producing the X-ray GRB tail and afterglow, we tend to believe that the expanding blast wave crosses a region of reduced density ambient gas, similar to what has been argued for the X-ray afterglow of GRB 981226, as reported by Frontera et al. (2000). Those authors conclude that this may point to a dying massive star scenario for the GRB progenitor. The data are insufficient to elaborate on this hypothesis as a viable origin scenario for GRB 990510, or to speculate further on the cause of the discrepancy between estimated upper limit and model prediction. (Note that shifting the initial time by more than $72 \mathrm{~s}$ with respect to trigger time allows recovering qualitative consistency of the first WFC upper limit with a single power-law from the start of the burst tail to the afterglow, but leads to exceedingly small $p$ values, and therefore does not allow a good fit of the overall multiwavelength afterglow.)

The X-ray spectral shape during the $80-130 \mathrm{~s}$ interval after the GRB, $\nu^{-1}$, suggests that, in a $\nu f_{\nu}$ representation, the peak energy is located within or just below the WFC band at this epoch. Since the physical parameters of the shock (see Fig. 1) imply that the transition between the fast and slow cooling occurs at $\lesssim 100$ s after the GRB (taking into account inverse Compton losses), this peak corresponds to the crossing of the injection $\left(\nu_{\mathrm{m}}\right)$ and cooling $\left(\nu_{\mathrm{C}}\right)$ frequencies. The $\nu_{\mathrm{C}}$ subsequently decreases, in a way approximately consistent with adiabatic or radiative evolution, reaching the far-ultraviolet band at the collimation break time $(\sim 1$ day after GRB), as inferred from the optical and X-ray spectral indices $\left(\beta_{\mathrm{op}} \simeq 0.6\right.$, Stanek et al. 1999; $\beta_{\mathrm{X}} \simeq 1.1$, KAK00), as predicted by PK00 (see their Fig. 3), and as computed empirically by us (Fig. 3b). Our values of $\nu_{\mathrm{C}}$ after the collimation break time are compatible with the predicted constancy (Sari et al. 1998; Sari et al. 1999), although they do not provide a strong proof (the error bars for $\nu_{\mathrm{C}}$ are sufficiently large to allow the $t^{-1 / 2}$ behavior expected for spherical afterglows. Moreover, if the electron cooling is inverse Compton dominated, then $\nu_{\mathrm{C}}$ may be flat in time).

\section{Conclusion}

In this paper we have focussed on the X-ray temporal behavior of GRB 990510 with the aim of confirming observationally the validity, at these frequencies, of a decelerating and sideways expanding jet emitting geometry. We have reached the following conclusions:

1) the X-ray afterglow of GRB 990510 can not be described by a single power-law, because the extrapolation of this model back to the time of the GRB is inconsistent with the latest X-ray data of the prompt event;

2) synchrotron radiation in a relativistic shock which propagates in a jet decelerating in a uniform external medium results in a continuously steepening temporal curve, which accounts well for the multiwavelength afterglow. The X-ray afterglow prediction at $\sim 100 \mathrm{~s}$ after trigger matches the last observed WFC point and is consistent with the WFC upper limits. Thus, not only GRB 990510 is the burst for which the clearest evidence of a spreading jet has been found in the optical afterglow, but it is also the unique case for which such evidence is present in the X-ray afterglow;

3) despite the good consistency noted in point (2) above, the temporal slopes of prompt event latest portion and afterglow differ by $\Delta \alpha \simeq 2.7$. Their similarity can be recovered if one assumes different physical initial times for the GRB and the afterglow;

4) in this last scenario, the first WFC upper limit still suggests a (not dramatic) deviation from a simple, monotonic behavior, which may be ascribed to ambient medium non-uniformity.

Future observations of multiwavelength prompt and delayed emission from GRBs with a better temporal coverage and sampling during the first two days after the burst than it is now affordable, will allow us to study in detail the jet phenomenon in these events.

Acknowledgements. E. Pian is grateful for hospitality at the Space Telescope Science Institute under the Visitor Program during completion of part of this work. The referee, Ralph Wijers, is gratefully acknowledged for his comments and suggestions, which led to important conclusions and contributed to improve the paper.

\section{References}

Arnaud, K. A. 1996, Astronomical Data Analysis Software and Systems V, ed. J. Jacoby, \& J. Barnes, ASP Conf. Ser., 101, 17

Beuermann, K., Hessman, F. V., Reinsch, K., et al. 1999, A\&A, $352, \mathrm{~L} 26$

Bloom, J. S. 2000, GRB Circular Notice ${ }^{1}$, No. 756

Briggs, M. 2000, Talk at the Conference "GRB in the Afterglow Era", held in Rome (Italy), 16-20 October

Burenin, R. A., Vikhlinin, A. A., Gilfanov, M. R., et al. 1999, A\&A, 344, L53

Cardelli, J. A., Clayton, G. C., \& Mathis, J. S. 1989, ApJ, 345, 245

Costa, E., Frontera, F., Heise, J., et al. 1997, Nature, 387, 783

Costa, E. 1999, A\&AS, 138, 425

Covino, S., Lazzati, D., Ghisellini, G., et al. 1999, A\&A, 348, L1

Frontera, F., Costa, E., Piro, L., et al. 1998, ApJ, 493, L67

Frontera, F., Amati, L., Costa, E., et al. 2000, ApJS, 127, 59

Fruchter, A. S., Ferguson, H., Pepper, J., et al. 1999, GRB Circ. Not., No. 386

Fruchter, A. S., Hook, R., \& Pian, E. 2000, GRB Circ. Not., No. 757

Fukugita, M., Shimasaku, K., \& Ichikawa, T. 1995, PASP, 107, 945

Giblin, T. W., van Paradijs, J., Kouveliotou, C., et al. 1999, ApJ, 524, L47

\footnotetext{
${ }^{1}$ http://gcn.gsfc.nasa.gov/gcn/gcn3_archive.html
} 
Harrison, F., Bloom, J. S., Frail, D. A., et al. 1999, ApJ, 523, Sari, R., Piran, T., \& Narayan, R. 1998, ApJ, 497, L17 L121

Sari, R., Piran, T., \& Halpern, J. P. 1999, ApJ, 519, L17

Israel, G. L., Marconi, G., Covino, S., et al. 1999, A\&A, 348, Sari, R., \& Piran, T. 1999, ApJ, 520, 641 L5

Stanek, K. Z., Garnavich, P. M., Kaluzny, J., Pych, W., \& Thompson, I. 1999, ApJ 522, L39

Jager, R., Mels, W. A., Brinkman, A. C., et al. 1997, A\&AS, 125,557

Kumar, P., \& Panaitescu, A. 2000, ApJ, 541, L51

Tkachenko, A. Yu., Terekhov, O. V., Sunyaev, R. A., et al. 2000, A\&A, 358, L41

Kuulkers, E., Antonelli, L. A., Kuiper, L., et al. 2000, ApJ, Vreeswijk, P. M., Fruchter, A. S., Kaper, L., et al. 2001, ApJ, 538, 638 (KAK00) 546,672

Nicastro, L., Amati, L., Antonelli, L. A., et al. 1999, A\&AS, Wei, D. M., \& Lu, T. 2000, A\&A, submitted 138,437

Panaitescu, A., \& Kumar, P. 2000, ApJ, submitted [astro-ph/0010257] (PK00)

Piro, L., Amati, L., Antonelli, L. A., et al. 1998, A\&A, 331, in 't Zand, J. J. M., Amati, L., Antonelli, L. A., et al. 1998, L41

Rhoads, J. E. 1999, ApJ, 525, 737 [astro-ph/0012007]

Wijers, R. A. M. J., Vreeswijk, P. M., Galama, T. J., et al. 1999, ApJ, 523, L33

ApJ, 505, L119 